\title{
Categorical Semantics of Linear Logic For All
}

\author{
Valeria de Paiva (paiva@parc.com) \\ Palo Alto Research Center \\ 3333 Coyote Hill Road \\ Palo Alto, CA 94304, USA
}

\section{Introduction}

This paper is a survey of results on categorical modeling of linear logic, oriented towards logicians, interested in proof theory, category theory and linear logic, but not experts in either of these. There are other such surveys (Maietti et al., 2005; Schalk, 2004; Mellies, 2002) available and hence there is a need to explain why another such. The explanantion is simple: I find the other surveys too hard to read, going too deeply into the details of definitions and proofs, when an interested, but not committed reader, would prefer to have intuitive explanations of which (and why) choices were made. Of course what is an intuitive explanation for some, will be considered glib glossing over by others. If I can convince my readers that the categorical modeling of the linear modality '!" is an interesting, albeit subtle, problem and why, this note will not have been a pointless exercise.

\section{The problem}

What are the requirements for a categorical model of linear logic? There are several solutions to this question in the literature. How do we compare and relate these solutions? That is, how do we compare and relate the several possible notions of 'categorical model of linear logic' available? This is the problem we want to address in this paper.

To solve this problem, first we must discuss what are categorical models, why should we bother with them and what are the requirements for a categorical model of linear logic in particular. Then we must explain why there are several solutions to this question and what these solutions are. Finally we must compare these solutions, pointing out the trade-offs between approaches.

The question "what is a modal of linear logic?" has been around for a while and there are many proposals on the table. For a while the best answer was the very clear paper of Bierman entitled exactly "What is

(C) 2006 Kluwer Academic Publishers. Printed in the Netherlands. 
a categorical model of intuitionistic linear logic?"(Bierman, 1994), but more work ensued and there is a need to incorporate the new work into a coherent framework. This has been done in a series of recent papers (Maietti et al., 2005; Mellies, 2002; Schalk, 2004) which aim at a categorically sophisticated audience. This paper attempts to explain to a practicing logician, in simple terms, using as little mathematics as possible, what is known about the categorical semantics of linear logic: as such it is a simplification of the work in (Maietti et al., 2005; Mellies, 2002; Schalk, 2004), which have the same goals, but are more technical, pressupose more knowledge of category theory and leave many of their conclusions to be worked out by the reader. Practicing logicians may brush up on their basic category theory in "Category Theory for Linear Logicians"(Blute and Scott, 2004), which has similar goals, and defines all categorical notions necessary for this paper. Note however that (Blute and Scott, 2004) avoids the discussion of what is required to deal the linear logic exponentials (cf.page 31), the main object of this paper, simply remarking that "the structure seems less canonical" .

\section{Categorical modelling: what and why}

There are two main uses of "categorical modeling": The first one considers the notion of a structure valued in a category $C$, the analogue of the classical model-theoretical notion of structure, except that one generalizes from (the category of) sets and functions to an abstract category $C$ with morphisms. This leads to what may be called "categorical model theory".

The second use, the one that concerns us here, is when we want to model more than theorems, we want to model different proofs of a given theorem. The idea, which has been called the "extended CurryHoward isomorphism", is that since we can associate terms in a typed lambda calculus with derivations in Natural Deduction and with morphisms in certain appropriate classes of categories, we can model these terms or "proofs"in the categories and we can then use equality of morphisms in the categories to induce a notion of equality of proofs in Natural Deduction for the logic in question. This is sometimes called "categorical proof theory". Applications of categorical proof theory include foundations of functional programming, proofs of correctness of compiler optimizations, new insights into expressive type systems, etc.

However, while the situation for categorical proof theory of (a Natural Deduction formulation of) intuitionistic propositional logic can be summarized in one sentence "categorical models of intuitionistic 
propositional logic are cartesian closed categories with coproducts", the situation for categorical proof theory of propositional linear logic is more complicated. In an ideal world one would define a special class of categories, perhaps linear categories (some sort of analogue of cartesian closed categories), prove that linear categories are sound and complete for linear logic and have one or two corollaries showing that other notions of model of linear logic in the literature are equivalent to (or subsumed by) this definition of a linear category. This kind of situation obtains for the modality-free fragment of intuitionistic linear logic, where the appropriate class of categories are symmetric monoidal closed categories (with products and coproducts to model additives). But the modalities pose some real problems. For the modality "!" (which Girard calls the exponential "of course!"), it is possible to prove soundness and completeness for several, in principle very different, notions of categorical model and the corollaries relating these different notions of model are confusing. Hence this note.

Before dealing with the complicated mathematics of the modality, let us first settle some minor issues. First, it is clear that even if one is interested in classical linear logic, producing the appropriate requirements for modeling intuitionistic linear logic is sufficient, as categorical duality will produce a model of classical linear logic from one of intuitionistic linear logic, automatically.

Secondly, the logic in question, presented as a sequent calculus by Girard and Lafont (Girard and Lafont, 1987) is pretty much uncontroversial. Part of the problem here is an adequate Natural Deduction formulation of the sequent calculus for intuitionistic linear logic (reproduced in the appendix) plus a term assignment for this Natural Deduction formulation satisfying some natural type-theoretical properties, like 'subject reduction', normalization, confluence, etc. The rest of the problem is how to phrase the categorical concepts in the simplest, more direct way possible.

Thirdly one could perhaps be tempted to 'ditch' the modality "!" (pronounced 'plink', 'shriek' or 'of course!') given the mathematical difficulties associated with it. This is not sensible, as it is the modality that makes Linear Logic so useful as a logical system. While Linear Logic is a resource sensitive logic, where one can pay attention to how many times resources like premisses are used, Girard's translation of usual (intuitionistic or classical) logic into Linear Logic allows us to forget this resource-consciousness, if desired. Thus not modeling the exponential is like throwing the baby away with the bathwater: since "!" is the way to recover classical logic expressivity in a linear setting, we must deal with it. The question is how to do it in the less painful way. 


\subsection{Preliminaries}

The fundamental idea of a categorical treatment of proof theory is that propositions should be interpreted as the objects of an appropriate category and (natural deduction) proofs of propositions should be interpreted as morphisms of that category. We use the traditional notational simplification of using the same name for a proof and its representation in the category. Fixing notation we say:

Definition 1. A category $\mathrm{C}$ is said to be a categorical model of a given logic $\mathcal{L}$, iff

1. For all proofs $\Gamma \vdash_{\mathcal{L}} M: A$ there is a morphim $[[M]]: \Gamma \rightarrow A$ in $\mathrm{C}$.

2. For all equalities $\Gamma \vdash_{\mathcal{L}} M=N$ : $A$ it is the case that $[[M]]={ }_{\mathrm{C}}[[N]]$, where $=_{\mathrm{C}}$ refers to equality of morphims in the category $\mathrm{C}$.

We say a notion of categorical model is complete if for any signature of the logic $\mathcal{L}$ there is a category $\mathrm{C}$ and an interpretation of the logic in the category such that:

If $\Gamma \vdash M: A$ and $\Gamma \vdash N: A$ are derivable in the system then $M$ and $N$ are interpreted as the same map $\Gamma \rightarrow A$ in the category $\mathrm{C}$ just when $M=N: A$ is provable from the equations of the typed equational logic defining $\mathcal{L}$.

As a reminder let us recall the categorical modeling of the fragment of intuitionistic linear logic consisting only of linear implications and tensor products, plus their identity, the constant $I$. (This fragment is sometimes called rudimentary linear logic.) For this fragment, the natural deduction formulation of the logic is uncontroversial. The rules for linear implication are just like the rules for implication in intuitionistic logic (with the understanding that variables always used a single time). The rules for tensor are slightly different from rules for usual cartesian product, as tensor products $A \otimes B$, unlike cartesian products $(A \times B)$, do not satisfy projections $(A \otimes B$ does not prove $A$ or $B)$ and do not allow for duplication ( $A$ does not prove $A \otimes A$ ). While such structures are less common that usual cartesian products, they are not uncommon in mathematics. Moreover, structures consisting of linear-like implications and tensor-like products had been named and investigated by category theorists decades earlier. They are called symmetric monoidal closed categories or smccs. Symmetric monoidal closed categories are soundand complete for rudimentary linear logic. 


\section{Modeling the Modality}

The original sequent rules for the modality '!' are intuitive. One should think of $! A$ as a formula which one is allowed to duplicate and erase at will, unlike plain $A$. These correspond to the usual rules of weakening and contraction:

$$
\frac{\Delta \vdash B}{\Delta, ! A \vdash B} \quad \frac{\Delta, ! A, ! A \vdash B}{\Delta, ! A \vdash B}
$$

But to make "!" a proper connective we must also introduce it to the right and to left of the turnstile. These rules are more complicated, but familiar from Prawitz's work on S4.

$$
\frac{! \Delta \vdash B}{! \Delta \vdash ! B} \quad \frac{\Delta, A \vdash B}{\Delta, ! A \vdash B}
$$

Now to transform the rules above into Natural Deduction ones with a sensible term assignment is not very hard. We obtain rules like:

$$
\begin{gathered}
\frac{\Delta \vdash M: ! A}{\Delta \vdash \operatorname{derelict}(M): A} \quad \frac{\Delta_{1} \vdash M: ! A \quad \Delta_{2} \vdash N: B}{\Delta_{1}, \Delta_{2} \vdash \operatorname{discard} M \text { in } N: B} \\
\frac{\Delta_{1} \vdash M: ! A \quad \Delta_{2}, a: ! A, b: ! A \vdash N: B}{\Delta_{1}, \Delta_{2} \vdash \operatorname{copy} M \text { as } a, b \text { in } N: B} \\
\frac{\Delta_{1} \vdash M_{1}: ! A_{1}, \ldots, \Delta_{k} \vdash M_{k}: ! A_{k} \quad a_{1}: ! A_{1}, \ldots, a_{k}: ! A_{k} \vdash N: B}{\Delta_{1}, \Delta_{2}, \ldots, \Delta_{k} \vdash \text { promote } M_{i} \text { for } a_{i} \text { in } N: ! B}
\end{gathered}
$$

The upshot of these rules is that each object ! $A$ has morphisms of the form er:! $A \rightarrow I$ and dupl: $! A \rightarrow ! A \otimes ! A$, which allow us to erase and duplicate the object !A. These morphisms give $! A$ the structure of a (commutative) comonoid.

Also each object ! $A$ has morphisms of the form eps: $! A \rightarrow A$ and delta:! $A \rightarrow ! ! A$ that provide it with a coalgebra structure, induced by a comonad. How should the comonad structure interact with the comonoid structure? This is where the picture becomes complicated and different choices can be made.

\subsection{LAFONT'S MODELS}

Even before Girard's original paper in Linear Logic appeared, Lafont had come up with a very elegant suggestion for a categorical model of intuitionistic linear logic. He suggested that the comonoids!A should be free. 
Definition 2. A Lafont category consists of

1. A symmetric monoidal closed category $\mathrm{C}$ with finite products,

2. For each object $A$ of $\mathrm{C}$, the object! $A$ is the free commutative comonoid generated by $A$.

Freeness (and co-freeness) of algebraic structures gives very elegant mathematics, but concrete models satisfying cofreeness are very hard to come by. None of the original models of Linear Logic satisfied this strong requirement ${ }^{1}$. Moreover, as remarked by Mellies, in categories of games researchers are interested in co-existing notions of '!', to model particular memory management paradigms, and if we use this notion of model there is only one cofree comonoid. But luckly there was already another proposed set of requeriments, that came to be known as the Seely model.

\subsection{SEELY'S MODELS}

Seely's notion of model(Seely, 1989) is much more encompassing, all models of linear logic (with additive conjunction) satisfy it. Instead of giving comonoids the prominent position they had in Lafont's definition, it takes the view, originally put forward by Girard, that linear logic is the basic logic and intuitionistic logic is to be considered a derived logic. This is because intuitionistic implication $A \rightarrow B$ can be decomposed into an use of the modality and one of linear implication, $A \rightarrow B={ }_{\text {def }}(! A) \multimap B$ via Girard's translation.

Seely's definition of a categorical model of intuitionistic linear logic requires the presence of additive conjunctions in the logic and it depends both on named natural isomorphims

$$
\mathrm{m}: ! A \otimes ! B \cong !(A \& B) \text { and } \mathrm{p}: 1 \cong ! \mathrm{T}
$$

and on the requirement that the functor part of the comonad '!' take the comonoid structure of the cartesian product to the comonoid structure of the tensor product. This notion of model had been in use for a few years, when, surprisingly, Bierman pointed out that Seely's definition missed one crucial condition. This meant that the comonoid structure of $! A$ and its comonad structure did not interact as well as needed to make it a sound categorical model. The condition missing can be simply added via what Bierman called a new-Seely category.

Definition 3 (Bierman). A new-Seely category, C, consists of

\footnotetext{
${ }^{1}$ The notable exception being dialectica categories (de Paiva, 1989).
} 
1. A symmetric monoidal closed category $\mathrm{C}$, with fite products, together with

2. A comonad $(!, \varepsilon, \delta)$ to models the modality, and

3. Two natural isomorphism, $\mathrm{n}: ! A \otimes ! B \cong !(A \& B)$ and $\mathrm{p}: I \cong ! \mathrm{T}$,

such that the adjunction between $\mathrm{C}$ and its co-Kleisli category is a monoidal adjunction.

Soundness of new-Seely categories is proved by showing that every new-Seely category is a linear category.

\subsection{Linear Categories}

Linear categories were introduced by Benton, Bierman, de Paiva and Hyland in (Benton et al., 1992) and carefully investigated by Bierman in his dissertation. The original definition of a linear category is a bit of a mouthfull, as it spells out the relationship between the comonoid and the comonad structure of $! A$ as fully as possible. It also unpacks into quite a collection of diagrams that need to be checked. But it does what it was meant to do and the soundness of the model is fully proved in (Bierman, 1994; Bierman, 1994).

Another ways of describing linear categories, championed by Hyland and Schalk(Hyland and Schalk, 1999) is to say that

Definition 4. A linear category is a symmetric monoidal closed category with products and equipped with a linear exponential comonad.

This is certainly easier to say, but to prove that it is indeed equivalent to linear categories as originally defined requires some work, done by Schalk in(Schalk, 2004).

Similarly, the following reformulation of the original definition was given by Maneggia in her thesis(Maneggia, 2004), where it is also proved equivalent to to the original definition.

Definition 5. A linear category is a symmetric monoidal closed category S together with a symmetric monoidal comonad such that the monoidal structure induced on the associated category of EilenbergMoore coalgebras is a finite product structure.

All the notions of model that we have seen so far agree on having a basis, which is a symmetric monoidal closed category $\mathrm{S}$ modeling the linear propositions. They also agree that there must be a functor !: S $\rightarrow$ S modeling the modality 'of course!'. They differ from each other on how they model the action of the modality functor. Basically linear categories (and new-Seely models) consider minimal conditions on 
some given monoidal comonad to make sure that the conditions guarantee the existence of an associated cartesian closed category modeling intuitionistic logic.

A different proposal came from ideas discussed independently by Benton, Plotkin and Barber. They suggested modeling the system more symmetrically, giving linear logic and intuitionistic logic the same status. This required introducing a new kind of term calculus, somewhat similar to Girard's logic of unity, where sequents have different kinds of contexts. But as far as the models are considered this is not such a big step. Having a monoidal comonad on a category $\mathrm{C}$ means that this comonad induces a spectrum of monoidal adjunctions spanning from the category of Eilenberg-Moore coalgebras to the co-Kleisli category. (This is basic category theory) Now putting linear logic and intuitionistic logic on the same footing, corresponds to saying that we decide to consider as the model of the system the monoidal adjunction itself, instead of the inducing comonad.

\subsection{Monoidal adjunctions}

Benton first suggested taking the monoidal adjuction (between a cartesian closed category $\mathrm{C}$ and a symmetric monoidal closed category $\mathrm{S}$ ) as the mathematical definition of model of the interaction between linear logic and intuitionistic logic. He called these kind of monoidal adjunctions $L N L$ models (for linear-non-linear models) and designed a term calculus corresponding to these models that he proved sound and complete.

This, more than simply a simplification of the notion of model of linear logic, corresponds to a shift of paradigms. While before one was trying to model a system of linear logic and it so happens that to model one of its connectives we needed a bridge to a different logical system, intuitionistic logic, now we are talking about modeling pairs of logical systems, with some important relationships between them. Moreover, since the whole of intuitionistic logic can be coded up inside linear logic via Girard's translation, in principle by modeling the systems side-byside we have a wholesale duplication of the intuitionistic proofs. This can be seen both as a good or a bad thing. In the one hand, one of the hopes for linear logic was that it would point out where duplication and erasing were necessary in specific proofs. In the LNL system this ability to pinpoint where duplication and erasing are required, disappears. But on the other hand, thinking of programming in the system, it allows you to keep and use all the libraries and optimizations that you may have created for intuitionistic logic previously. Moreover, the definition of a LNL model is so simple it almost does not require a 
Definition 6. A linear-non-linear (LNL) category consists of a symmetric monoidal closed category $\mathrm{S}$, a cartesian closed category $\mathrm{C}$ and a symmetric monoidal adjucntion between them.

Benton also proved that linear categories and LNL models are equivalent, in the sense that given a LNL model one obtains a linear category and conversely, given a linear category you can obtain a LNL model. But there is no uniqueness of the categories obtained.

Barber and Plotkin improved on Benton's suggestion, by showing that one does not need to require closedness of the cartesian category. Function spaces in the cartesian category can be induced from the linear ones. They also produced a term calculus, called DILL for Dual Intuitionistic and Linear Logic, that looks much more like intuitionistic linear logic that LNL and satisfies all essential syntactic properties of LNL. To model DILL they considered the notion of a DILL-category, which differs from a LNL-category only on not requiring a cartesian closed structure to be given a priori. You only need to start with a cartesian structure and you then prove it cartesian closed.

Definition 7. $A$ dual intuitionistic linear logic (DILL) category is a symmetric monoidal adjunction between $\mathrm{S}$ a symmetric monoidal closed category and $\mathrm{C}$ a cartesian category.

By now we have one definition of a categorical model of a generic logic $\mathcal{L}$ and six slightly different definitions of a model of linear logic. How do they compare? Which one is best? Why?

\section{Comparing Models}

As discussed before we know that Lafont models are very specific instances of linear categories. We also know that new-Seely categories are linear categories. Further we know that Hyland and Schalk's symmetric monoidal categories with a linear exponential comonad are linear categories. Also Benton and Mellies have proved, independently, that given a linear category we obtain a DILL-category and given a DILLcategory we obtain a linear category. Are all these notions of model equivalent then?

Maietti, Maneggia, de Paiva and Ritter (Maietti et al., 2005) set out to prove some kind of categorical equivalence of models and discovered that the situation was not quite as straightforward as expected. To wit: Bierman proved that linear categories are sound and complete with respect to ILL. Barber proved that DILL-categories are sound and complete with respect to DILL. Maietti et al proved (theorem 
18 in (Maietti et al., 2005)) that the category of theories of ILL is equivalent to the category of theories of DILL. So we have two calculi, ILL and DILL, sound and complete with respect to their models, whose categories of theories are equivalent. One would expect their categories of models (linear categories and symmetric monoidal adjunctions) to be equivalent too. But when we consider the natural morphisms of linear categories and of symmetric monoidal adjunctions (to construct categories Lin and SMA), we do not obtain a categorical equivalence. Instead we obtain only a retraction (theorem 41 in (Maietti et al., 2005)). So we faced with the somewhat paradoxical situation of calculi with equivalent categories of theories, whose classes of model are not equivalent.

This led Maietti et al to conclude that soundness and completeness of a notion of categorical model are not enough to determine the "most apropriate" notion of categorical model for a calculus. More than simply soundness and completeness we should say a class of categories is a model for a type theory when we can prove an internal language theorem relating the category of models to the category of theories of the calculus.

What does it mean to prove an internal language theorem for a typed calculus and a class of categorical models? Given a type calculus $\mathcal{L}$, one can consider its category of theories, $\operatorname{Th}(\mathcal{L})$ and its category of sound and complete models, together with model morphisms $\mathcal{M}(\mathcal{L})$. We say that a typed calculus provides an internal language for the class of models in $\mathcal{M}(\mathcal{L})$ if we can establish an equivalence of categories between the category of $\mathcal{L}$-theories, $\operatorname{Th}(\mathcal{L})$ and the category of $\mathcal{L}$-models $\mathcal{M}(\mathcal{L})$. The functors establishing this equivalence, say, $L: \mathcal{M}(\mathcal{L}) \rightarrow \operatorname{Th}(\mathcal{L})$ and $C: \operatorname{Th}(\mathcal{L}) \rightarrow \mathcal{M}(\mathcal{L})$ allow us to move freely between categorical syntax and categorical semantics. So $L$ assigns to a model $M$ in $\mathcal{M}(\mathcal{L})$ its specific internal language $L(M)$. While the functor $C$ associates to each specific $\mathcal{L}$-theory $V$ its syntactic category $C(V)$ in such way that $M \cong C(L(M))$ and $V \cong L(C(V))$.

We note that this definition of internal language is more restrictive than the one (in Barr and Wells for example) which requires only one of the equivalences, namely $M \cong C(L(M))$ to hold.

Tightening their definitions as above, Maietti et al obtain the desired situation that equivalent calculi (DILL and ILL), with equivalent categories of theories (Th(DILL) $\cong T h(I L L)$ ) have equivalent classes of models. But this necessitates postulating that despite being sound and complete for DILL, symmetric monoidal adjunctions between a cartesian and a symmetric monoidal closed category (the category SMA) are not the models for DILL. Instead we must take as models for DILL a subcategory of SMA, the symmetric monoidal adjunctions generated 
by finite tensor products of free coalgebras. (This idea originally due to Hyland, was expanded on and explained by Benton and Maietti et al.)

With hindsight one can see that the reason why the calculus DILL does not provide an internal language for all the natural symmetric monoidal adjunctions is that the cartesian category of a given symmetric monoidal adjunction (S, C, $F \dashv G$ ) may have objects and morphisms that are not in the domain of functor $F$ and hence no DILL-theory can provide syntax corresponding to these objects and morphisms. They are genuinely not part of the linear-non-linear picture.

Thus the price to pay for the expected result that equivalent categories of theories imply equivalent categories of models is high: not only we have to keep the more complicated notion of model of linear logic, but we need also to insist that categorical modeling requires soundness, completeness and internal language theorems. While this last requirement makes sense in general, it comes about in this setting from a syntactic perspective, one that enforces very restrictive notions of morphisms of linear categories. Hyland and Schalk, coming from a perspective less syntactic, geared towards categories of games, came up with a more relaxed notion of linear morphism.

Definition 8. Let $\mathrm{C}$ and $\mathrm{D}$ be two linear categories. A functor $F: \mathrm{C} \rightarrow$ $\mathrm{D}$ is linearly distributive if and only if $F$ is monoidal and is equipped with a distributive law $\kappa: ! F \rightarrow F$ ! respecting the comonoid structure.

Maietti et al show that their more strict notion of linear functor is actually a linearly distributive functor as above.

A slightly different approach to carving out the relevant part of the category of symmetric monoidal adjunctions is taken by Møgelberg, Birkedal and Petersen(Mgelberg et al., 2005). They call linear adjunctions the symmetric monoidal adjunctions between an smcc and a cartesian category and say that DILL-models are the full subcategory of the category of linear adjunctions on objects equivalent to the objects induced by linear categories, when performing the product of free coalgebras construction.

A very different semantic approach to putting together a linear-nonlinear logic is pursued by Maietti, de Paiva and Ritter(Maietti et al., 2000), who following the lead of Plotkin and Wadler, consider a calculus (called ILT for intuitionistic and linear type theory) without the modality '!', only with interaction between a linear and an intuitionistic implication. Comparison between that approach and the one in this note is left to future work. 


\subsection{Models With PRODUCTS}

So far we discussed models of a calculus consisting of only tensor products, linear implications and modalities '!'. If our calculus also has finite cartesian products (or additive conjunctions) the mathematical structures simplify considerably. The point is that a linear category with products yields a co-Kliesli category with products and this is actually cartesian closed. This makes it easy to show that models of ILL with products and of DILL with products are equivalent, as expected (proposition 54 in (Maietti et al., 2005)). Somewhat surprisingly, given that DILL was invented from LNL, Maiettti et al also show that models of DILL with products are only a subcategory of models of LNL with products.

\section{Conclusions}

We surveyed several notions of categorical model of intuitionistic linear logic and compared them, as categories. The main comparison was between linear categories (in its various guises) and symmetric monoidal adjunctions as proposed by Benton, Barber and Plotkin. The summary is that while the notion of a symmetric monoidal adjunction (between a symmetric monoidal closed category and a cartesian category) is very elegant and appealing, the category of these SMA is too big, has objects and morphisms that do not correspond to objects and morphisms in dual intuitionistic and linear logic. We also tried to explain the point, made by Maietti et al(Maietti et al., 2005), that categorical modeling should be about soundness, completeness and essentially internal language theorems. While we agree that this extra criterion is a sensible one, we feel that the only kind of evidence provided (that without it, we have one example of two calculi ILL and DILL with equivalent theories that do not have equivalent classes of models) is not strong enough. A traditional logician may prefer to think that the problem we discussed is a problem of intuitionistic linear logic, which is not as well-behaved as it should be. This is not our conclusion. The problem with modeling the modality 'of course!' of linear logic is similar to the problem of modeling any other modalities and these are pervasive in logic. More research into other modalities should clarify the criteria for categorical modeling in general. Also new concrete mathematical models, like games, for other logical systems, should help. 


\section{References}

Benton, N., G. Bierman, V. de Paiva, and M. Hyland: 1992, 'Term Assignment for Intuitionistic Linear Logic.'. Technical report, University of Cambridge.

Bierman, G.: 1994, 'On Intuitionistic Linear Logic'. Technical Report 346, Computer Laboratory, University of Cambridge. Ph.D. Thesis.

Bierman, G.: 1994, 'What is a Categorical Model of Intuitionistic Linear Logic?'. In: Proc. of the Second International Conference on Typed Lambda Calculus and Applications., Vol. 902 of Lecture Notes in Computer Science. Springer Verlag.

Blute, R. and P. Scott: 2004, 'Category Theory for Linear Logicians.'. In: C. U. Press (ed.): Linear Logic in Computer Science.

de Paiva, V.: 1989, 'The Dialectica Categories.'. In: Categories in Computer Science and Logic., Vol. 92 of Contemporary Mathematics. pp. 47-62, American Mathematical Society.

Girard, J. Y. and Y. Lafont: 1987, 'Linear Logic and lazy computation.'. In: TAPSOFT '87, Vol. 250 of Lecture Notes in Computer Science. pp. 52-66.

Hyland, M. and A. Schalk: 1999, 'Abstract Games for Linear Logic. Extended Abstract'. In: CTCS '99, Vol. 29 of Electronic Notes in Theoretical Computer Science.

Maietti, M. E., V. de Paiva, and E. Ritter: 2000, 'Categorical Models for Intuitionistic and Linear Type Theory.'. In: J. Tiuryn (ed.): Proc. of Foundations of Software Science and Computation Structures.

Maietti, M. E., P. Mannegia, V. de Paiva, and E. Ritter: 2005, 'Relating Categorical Models for Intuitionistic Linear Logic.'. Applied Categorical Structures 13, 1-36.

Maneggia, P.: 2004, 'Models of Linear Polymorphism'. Ph.D. thesis, The University of Birmingham, UK.

Mellies, P. A.: 2002, 'Categorical models of linear logic revisited.'. submitted to Theoretical Computer Science.

Mgelberg, R., L. Birkedal, and R. Petersen: February 2005, 'Categorical models of PILL.'. Technical Report TR-2005-58, IT University of Copenhagen.

Schalk, A.: 2004, 'What is a Categorical Model of Linear Logic?'. University of Manchester. http://www.cs.man.ac.uk/ schalk/notes/index.html.

Seely, R. A. G.: 1989, 'Linear logic, *-autonomous categories and cofree coalgebras.'. In: Categories in computer science and logic (Boulder, CO, 1987), Vol. 92 of Contemp. Math. pp. 371-382, Amer. Math. Soc., Providence. 